\title{
Progress in Diagnosis and Treatment of Upper Gastrointestinal Hemorrhage
}

\author{
Ruizhi Hou, Yan Liu \\ China-Japan Union Hospital of Jilin University, Changchun, Jilin, 130021, China
}

Keywords: Upper gastrointestinal hemorrhage, Diagnostic study, Treatment progress, Research significance

\begin{abstract}
Upper gastrointestinal hemorrhage refers to the blooding of the upper gastrointestinal tract, including esophagus, stomach and duodenum, which is located above the flexor ligament. Although it is a common disease in clinic, and with the development of medical technology, the treatment method is improving continuously. However, it has the characteristics of fast onset and complicated condition, and it has important prognostic significance for the timely diagnosis and treatment of the disease. This paper would summarize the new progress of clinical diagnosis and treatment of upper gastrointestinal hemorrhage in recent years, and further explain the etiology and clinical symptoms of upper gastrointestinal hemorrhage, so as to provide reference for clinical diagnosis of upper gastrointestinal hemorrhage and improve the effect of treatment of upper digestive tract hemorrhage.
\end{abstract}

\section{Introduction}

Acute upper gastrointestinal hemorrhage refers to the acute hemorrhage caused by lesions of the digestive organs above the flexor ligament, as well as the upper jejunum hemorrhage after gastrojejunostomy. The disease is one of the most common clinical emergencies with a mortality of 8 percent to 13.7 percent. Hematemesis and hematochezia are the main clinical manifestations, or there is the gastroscopic hemorrhageing fluid in the stomach. Due to the progress of modern clinical diagnosis and treatment technology, the mortality rate of this disease has been controlled below 10 percent, which is significantly lower than before. However, the condition of this disease is very dangerous, so we must strictly deal with it according to certain procedures and steps in the treatment. In addition to the use of internal medicine conservative treatment measures, and according to the actual condition of the disease and the patient, we can combine the surgical operation with endoscopic hemostasis. The progress of diagnosis and treatment of this disease is summarized as follows.

\section{Etiology of Upper Gastrointestinal Hemorrhage}

There are many causes of upper gastrointestinal hemorrhage, such as peptic ulcer, acute gastric mucosal lesions, esophageal and gastric varices and gastric cancer. There are three main causes: systemic diseases, esophageal and gastric varices, and related causes of non-varicose veins. Cardiovascular and cerebrovascular diseases, blood diseases and other coagulation mechanism disorders may lead to upper gastrointestinal hemorrhageing; the rupture of esophageal and gastric varices was mainly due to cirrhosis and hemorrhage. However, most of that upper gastrointestinal tract hemorrhage caused by non-varsity is caused by digestive tract disease. In recent years, the incidence of gastric mucosal erosion has increased with the use of non-body anti-inflammatory drugs, and the number of cases of upper gastrointestinal hemorrhageing caused by it has increased year by year.

\section{Clinical Symptoms of Upper Gastrointestinal Hemorrhage}

The symptoms of upper gastrointestinal hemorrhage are obvious, which is usually accompanied by black stool and hematemesis. Patients with acute upper gastrointestinal hemorrhage will appear 
the symptoms including hemorrhage dizziness, panic, cold limbs, faster pulse, lower blood pressure and other symptoms due to a large amount of hemorrhageing in a short period of time. Fainting or even shock may occur when the patient is standing. Some of the body parameters of the patients will also change due to hemorrhageing, and the number of reticulocyte increased within 24 hours after hemorrhageing. Moreover, the blood urea content increased significantly, and reached the peak within 24 to 48 hours and 3 to 4 days can return to normal level. According to the amount of blood loss in the upper digestive tract, the patient may develop hemorrhagic anemia after 3 to 4 hours. A large amount of hemorrhageing can lead to low fever and last for 3 to 5 days.

\section{Study on the Diagnosis of Upper Gastrointestinal Hemorrhage}

\subsection{Haemorrhage Characteristics}

If the patient has long-term hematemesis, hematochezia, anemia and other clinical symptoms, they need to be clinically diagnosed. First, the condition of the esophagus and stomach fundus hemorrhage is usually acute. If the amount of blood loss is large and reach to 650 to 950ml, the patient often appears body stress reaction after hematemesis, leading to shock. Therefore, the condition is more serious. Second, it is the duodenal hemorrhage: The volume of single hemorrhage at this site is usually 400 to $500 \mathrm{ml}$, and the patient does not generally develop a shock response. The main symptoms of the disease are persistent hematemesis and hematochezia. Third, hemobilia appeared slowly. Its hemorrhageing amount is less, which is generally in $250 \mathrm{ml}$ or so, and clinical manifestations are mainly hematochezia. Shock is rarely appeared.

\subsection{Amount of Hemorrhage}

The fecal blood volume of patients with acute upper gastrointestinal hemorrhage should be more than $5 \mathrm{ml} / \mathrm{d}$, and the body reaction of patients with different hemorrhage volume was also different. If the stool color of the patient is darker, the amount of hemorrhage is less than $60 \mathrm{ml}$. If the stool is tar like, the hemorrhage in the stomach has been over 7 hours. The symptoms of hematemesis showed that the total blood volume in the stomach was more than $300 \mathrm{ml}$, and the patient had symptoms of dizziness, dryness of mouth and illusory symptoms, indicating that the amount of hemorrhage lost was more than $420 \mathrm{ml}$. If the patient developed shock and syncope, the amount of blood loss was greater than $2000 \mathrm{ml}$, and urgent treatment was needed to prevent death caused by massive gastric hemorrhage.

\subsection{Pulse}

Pulse change is also one of the main methods for diagnosis of acute upper gastrointestinal hemorrhage. If the pulse of the patient is weak and the speed is more than 100 times/min, the amount of hemorrhage can be determined in the range of 800 to $1500 \mathrm{ml}$. If the patient maintains sitting posture or half-lying position there are dizziness, panic, night sweat and other symptoms, indicating a large amount of blood loss. Because of the special condition of some patients, it is very difficult to judge their condition through pulse. At this time, medical staff are required to use professional instruments and equipment. The central venous pressure was measured.

\subsection{Blood Pressure}

When the hemorrhage volume of patients with acute upper gastrointestinal hemorrhage is about $800 \mathrm{ml}$, there is no obvious abnormal blood pressure; moreover, the systolic blood pressure is slightly increased, and pulse pressure is decreased, so it is necessary to observe the changes of blood pressure in the patients and prevent shock. When blood loss was 800 to $1600 \mathrm{ml}$, systolic blood pressure was $75 \mathrm{~mm} \mathrm{Hg}$; when the blood loss is greater than $1600 \mathrm{ml}$, the systolic blood pressure is $60 \mathrm{~mm} \mathrm{Hg}$; moreover, the blood loss exceeds the total blood volume of the human body, and the blood pressure drops to zero. In addition to monitoring the patient's blood pressure changes, they also need to do bowel sound examination and anal examination. 


\section{Diagnosis and Study of the Etiology of Upper Gastrointestinal Hemorrhage}

Clinical symptoms and laboratory tests: To determine accurately whether the hemorrhage has stopped is the basis of the choice of treatment methods. The body manifestations of active hemorrhageing are as follows: First, it includes the number of symptoms such as hematemesis and hematochezia increased, time interval shortened, hemorrhageing color being dark and accompanied by very obvious bowel sounds. Second, after intravenous injection, the circulatory tissues of patients did not change, or there was a temporary improvement. The overall trend of change was worsening. After measuring central venous pressure, blood pressure showed instability, accompanying high and low and changed greatly. Third, hemoglobin count decreased, and reticulocyte count increased, and the change was obvious. It was found that the blood color of stomach was bright red and the blood urea content was increasing under the condition of sufficient urine volume.

Endoscopic examination: Endoscopic examination is characterized by high safety and simple operation. Patients do not generally have adverse reactions or complications. However, it is important to note that: Patients with severe cardiopulmonary dysfunction or patients who are unable to perform organ perforation cannot be examined by endoscopy and can be examined by traditional laparotomy. Endoscopic examination should be carried out quickly after the patient's condition is stable, and the appropriate time should be chosen to prevent the occurrence of various complications. Patients with shock caused by excessive blood loss should be examined after their vital signs such as blood pressure heart rate and other vital signs are stable. The diagnosis effect of endoscopic examination is remarkable and the hemorrhageing causes of patients can be detected more accurately. It has high clinical value.

\section{Research Progress of Pharmacologic Treatment}

\subsection{Progress in the Treatment of Esophageal and Gastric Varices Hemorrhage}

About 60 percent patients with cirrhosis and portal hypertension suffered from upper gastrointestinal hemorrhage due to rupture of esophageal and gastric fundus varices. At present, drug therapy is still the first choice for the treatment of esophageal variceal hemorrhage, because of its advantages including convenience, rapid completion, less adverse reactions, low price and etc. Vasoactive drugs and pituitrin should be given as early as possible to stop hemorrhageing by reducing portal blood flow and portal pulse pressure. First, it is vasopressin and its derivatives: It reduces the pressure of the portal vein of the liver by constricting the visceral blood vessels of the patient to reduce the visceral blood flow, so as to achieve the purpose of hemostasis. Although more adverse reactions, such as elevated blood pressure, angina pectoris, but it is still the first choice for the treatment of esophageal variceal hemorrhage. Second, it is the vasoconstrictors: By reducing blood flow through vasoconstriction, the esophageal myometrium can be constricted and the hemorrhageing of esophageal and gastric fundus varices can be alleviated. Third, it is the somatostatin: It can selectively reduce the effective visceral circulation blood flow, leading to the reduction of portal vein pressure. As a gastrointestinal hormone, it not only improves microcirculation to promote the repair of injured tissue mucosa, but also has no obvious effect on blood pressure of the whole body. Patients with esophageal variceal hemorrhage were treated with vasoconstrictor and somatostatin. The results showed that the hemostatic rates of these two drugs were similar. However, in terms of complications, somatostatin-induced complications were significantly less than vasoconstrictors. Fourth, it is the calcium channel blockers: It stops hemorrhageing by preventing calcium from entering the cell membrane, reducing vasoconstriction and reducing resistance to the portal vein.

\subsection{Progress in Drug Treatment of Non-varicose Upper Gastrointestinal Hemorrhage}

Peptic ulcer, upper digestive tract tumor and stress ulcer are the most common causes of non-varicose upper gastrointestinal hemorrhage. For patients with no obvious taboos, hemostasis should be treated under endoscopy. It is the gastric motility drugs: Before gastroscopy examination, 
the use of gastric motility drugs is beneficial to emptying intragastric hemorrhage, making the image of gastroscopy more clear and improving the accuracy of diagnosis. The reexamination rate of gastroscope was also significantly decreased, and the number of hospital day and the rate of operation were not significantly affected. First, it is the proton pump inhibitors: These drugs have strong acid suppression, fast onset, long duration and no drug tolerance. It is often recommended for large doses, significantly reducing the rate of rehemorrhageing and reducing the possibility of surgery. Proton pump inhibitors before endoscopy can significantly reduce the risk of endoscopic therapy in addition to reducing the risk of endoscopic treatment. Second, it is the receptor blockers: Its effect on acid suppression is very limited, and it is difficult to ensure that the stomach is less than 9 to the neutral level. Therefore, it is generally not recommended for routine use, but only for low risk patients. Third, it is the vasoactive drugs and somatostatin drugs: Both can reduce visceral blood flow by constricting visceral blood vessels, inhibit gastric acid secretion, and protect gastric mucosa. They can be used for liver cirrhosis upper gastrointestinal hemorrhage and non-varicose upper gastrointestinal hemorrhage. It has better curative effect. Fourth, prothrombin complex is the main coagulation factor used at present and can be supplemented with fresh dried plasma.

\section{References}

[1] Zhou Biao, Niu Xuping. New Progress in Diagnosis and Treatment of Upper Gastrointestinal Hemorrhage [J]. A Modern Chinese Doctor, 2009, 47(19): 31-34.

[2] Wang Fangyu, Shi Hui. Progress in Drug Treatment of Upper Gastrointestinal Hemorrhage in the elderly [J]. Practical Elderly Medicine, 2015, 29(03): 184-188.

[3] Huang Ping. Analysis on Diagnosis and Treatment of Upper Gastrointestinal Hemorrhage [J]. The Latest Medical Information Abstracts of the World, 2015, 15(51): 22-23.

[4] Li Jie. Progress in Diagnosis and Clinical Treatment of Acute Upper Gastrointestinal Hemorrhage [J]. Medical Theory and Practice, 2016, 29(22): 3056-3058.

[5] Luo Guihong. Progress in Diagnosis and Treatment of Intractable Gastrointestinal Hemorrhage [J]. Chinese Medical Guide, 2012, 10 (19): 105-107. 